\title{
ERRATUM
}

J Korean Soc Appl Biol Chem (2012) 55, 397-405

DOI $10.1007 / \mathrm{s} 13765-012-1924-9$

\section{Erratum to: Mango (Mangiferaindica L.) Peel Extracts Inhibit Proliferation of HeLa Human Cervical Carcinoma Cell via Induction of Apoptosis}

\author{
Md. Ramjan Ali · Moon Jeong Yong • Rajendra Gyawali • Ashik Mosaddik • \\ Youn Chul Ryu · Somi Kim Cho
}

Published online: 30 June 2012

(C) Springer-Verlag 2012

Table 4, which is missing in the paper, is given below.

The online version of the original article can be found under doi:10.1007/s13765-012-1024-x

Md. R. Ali and M. J. Yong contributed equally.

Md. R. Ali · M. J. Yong · Y. C. Ryu $\cdot$ S. Kim Cho $(\bowtie)$

Faculty of Biotechnology, College of Applied Life Sciences, Jeju National University, Jeju 690-756, Republic of Korea

E-mail:somikim@jejunu.ac.kr, phd.kim.somi@gmail.com

Md. R.Ali

Department of Biochemistry and Biotechnology, Khwaja Yunus Ali University, Sirajganj, Bangladesh

R. Gyawali · S. Kim Cho

Subtropical Horticulture Research Institute, Jeju National University, Jeju 690-756, Republic of Korea

A. Mosaddik

Department of Pharmacy, Rajshahi University, Bangladesh 6205 
Table 4 Compounds of methanolic extracts of Fozli peel tentatively identified by GC-MS

\begin{tabular}{|c|c|c|c|}
\hline Peak number & $\mathrm{RI}^{\mathrm{a}}$ & Compound $^{\mathrm{b}}$ & $\begin{array}{c}\text { Area } \\
\%^{c}\end{array}$ \\
\hline 1 & 1139 & 2,3-Dihydro-3,5-dihydroxy-6-methyl-4H-pyran-4-one & 6.43 \\
\hline 2 & 1236 & 5-Hydroxymethyl-2-furancarboxaldehyde & 17.20 \\
\hline 3 & 1383 & 2,5-Dihydroxyphenol & 22.72 \\
\hline 4 & 1858 & Hexadecanol & 4.41 \\
\hline 5 & 1919 & Hexadecanoic acid methyl ester & 0.49 \\
\hline 6 & 1959 & Pentadecanoic acid & 12.78 \\
\hline 7 & 2091 & $(Z, Z)-9,12$-Octadecadienoic acid methyl ester & 0.35 \\
\hline 8 & 2098 & (Z)-9-Octadecenoic acid methyl ester & 0.30 \\
\hline 9 & 2108 & Phytol & 1.00 \\
\hline 10 & 2131 & $(Z, Z)-9,12$-Octadecadienoic acid ethyl ester & 5.18 \\
\hline 11 & 2136 & $(Z, Z)-9,12$-Octadecadienoic acid & 8.79 \\
\hline 12 & 2155 & (Z)-9-Octadecenoic acid & 0.73 \\
\hline 13 & 2299 & Tricosane & 0.17 \\
\hline 14 & 2399 & Tetracosane & 0.34 \\
\hline 15 & 2499 & Pentacosane & 0.53 \\
\hline 16 & 2599 & Heptacosane & 0.52 \\
\hline 17 & 2800 & Octacosane & 1.13 \\
\hline 18 & 2900 & Nonacosane & 1.12 \\
\hline 19 & 3000 & Tricontane & 1.95 \\
\hline 20 & $3000<$ & $\alpha$-Tocopherol & 0.24 \\
\hline 21 & $3000<$ & Stigmasterol & 0.43 \\
\hline \multirow[t]{2}{*}{22} & $3000<$ & $\gamma$-Sitosterol & 1.31 \\
\hline & & Total & 88.12 \\
\hline
\end{tabular}

${ }^{a}$ Retention indices relative to $\mathrm{C}_{7}-\mathrm{C}_{30} n$-alkanes calculated on Rtx-5MS capillary column.

${ }^{\mathrm{b}}$ Compounds tentatively identified based on retention index and elution order as well as the fragmentation pattern described in the literature.

${ }^{c}$ Relative peak area percentage (peak area relative to the total peak area \%) 